\title{
Legal Medical Age Estimation in Portuguese Adult Cadavers: Evaluation of the Accuracy of Forensic Dental Invasive and Non-Invasive Methods
}

\author{
Pereira $\mathrm{CP}^{* 1,2,3}$, Caldas $\mathrm{R}^{2}$ and Pestana $\mathrm{D}^{1,4}$ \\ ${ }^{1}$ Center of Statistics and Applications of University of Lisbon, Portugal \\ ${ }^{2}$ Faculty of Dentistry, University of Lisbon, Portugal \\ ${ }^{3}$ CENCIFOR, Forensic Science Center, National Institute of Legal Medicine, Portugal \\ ${ }^{4}$ Department of Statistics and Operation Research, Faculty of Sciences, University of Lisbon, Portugal
}

${ }^{*}$ Corresponding author: Pereira CP, Assistant Professor, National Institute of Legal Medicine and Forensic Sciences, Delegation of South, Rua Manuel Bento de Sousa 3, 1169-201 Lisbon, Portugal, Tel: 00351 217804563, E-mail: cristiana.pereira@fmd.ul.pt

Citation: Pereira CP, Caldas R, Pestana D (2013) Legal Medical Age Estimation in Portuguese Adult Cadavers: Evaluation of the Accuracy of Forensic Dental Invasive and Non-Invasive Methods. J Forensic Sci Criminol 1(2): 201. doi: 10.15744/2348-9804.1.201

Received Date: June 19, 2013 Accepted Date: October 15, 2013 Published Date: October 24, 2013

\begin{abstract}
Age estimation of unknown human cadavers is important in setting a crime investigation, such as homicide, suicide or in mass disaster because it can guide forensic investigators to the correct identity among a large number of possible matches. More and more the courts ask for medico-legal age estimations of unknown human cadavers by forensic dentistry. This research project in establishing the applicability of radiographic and morphological parameter's used in forensic dentistry in a Portuguese adult population. The main goal is to compare the chronological age with the dental age estimation by radiographic and morphological parameters. To achieve this objective, thirty-five single-rooted teeth were analysed. Radiographic analysis was made through the program of digital radiology software Kodak RVG intra-oral 2200 with exposure factors $60-70 \mathrm{KVp}$ and $7 \mathrm{~mA}$. The morphological parameter, translucent dentin was assessed in intact and sectioned tooth. The relationship between the coefficients was calculated between the age and the ratios with the level of inclusion, $\mathrm{p}<0.05$. There was no statistically significant difference between the chronological age and the age estimation by each method. The coefficient of Pearson for the direct measurements of the translucent dentin methodology for sectioned teeth gives the better correlation with the age estimate $(\mathrm{r}=0.86)$.
\end{abstract}

\section{Keywords}

Forensic dentistry; Forensic pathology; Human identification; Biological age estimation; Dental age estimation; Portuguese adult cadavers

\section{Introduction}

\section{Age estimation in cadavers by forensic dentistry}

Actually there are a number of legal medical reasons for age estimation of an individual [1-3]. Age estimation is one of the main fields of forensic dentistry. The use of teeth in legal medical age estimation is an international forensic methodology accepted for cadaver identification due to their advantages [4]. The dental hard tissues, mainly the enamel, are the anatomical elements with the highest resistance to post mortem changes caused by humidity, high temperatures, microbiological activity and mechanical forces during the post mortem interval [5]. The teeth from cadavers may remain intact for the longest period of time without any macroscopic changes, microbial and chemical diagenetic factors, and furthermore their analysis is easier than bones [6]. Finally, another advantage of the teeth is that the results of age estimation by their mineralization and eruption are more accurate than provided by skeletal development [7]. With the end of these physiologic processes, the teeth are still the best evidence [7].

The dental age estimation has become an established practice in legal medicine and forensic dentistry. The forensic analysis of the cadaver can be done through different methods: morphological, radiological, biochemical, histological among others [3]. However there is no methodology that is $100 \%$ accurate in dental age estimation. The main reasons are systematic errors inherent of each scientific method for age estimation, the inter- and intra-individual variations of observers of each method and the factors dependent on the population in study such as, the inter- and intra-individual variability of growth due to ethnic, socio-economic and pathological factors [8]. 


\section{The methods of dental age estimation for this re- search project at an adult Portuguese population}

Radiograph Method: Kvaal and Solheim, presented for the first time, a methodology which relied on morphological and radiological measurements [6]. The regression formulas have been calculated for dental age estimation based on analysis of 6 teeth, 3 from upper jaw and 3 from lower jaw, based on analysis of each individual tooth [8]. The coefficient of regression analysis is higher when the ratios of 6 types of teeth, from upper and lower jaws are used. This coefficient decreased when teeth from the same jaw were used (or upper jaw or lower jaw) and became weaker when carried out measurements on lower canines [8]. The dental age estimation was established by 5 regression formulas for each dental group. The independent variables are pulp chamber sized when measured in the radiographies, the periodontal ligament retraction and the translucent dentin in the apical portion of root [6].

Direct method: Bang and Ramm has developed for the first time, a methodology using the measurement of the translucent dentin length at apical root and thus established a correlation with age [9]. The translucent dentin appears in the third decade of individual life progressing over time from the apical to the coronal direction in the root [10]. In the dentinal tubules occurs the deposition of a dense matrix of hydroxyapatite crystal which leads to the reduction of intra-tubular diameter [5]. The difference between the refractive indices of organic and inorganic material of the dentinal tubules increases the degree of translucent dentin [11]. This parameter is less influenced by environmental and pathological factors, when compared with others dental morphological changes [10].

The analysis was based on a set of regression equations for complete and sectioned teeth according to the tooth type and the length of the root translucent dentin in millimeters [9]. These authors proposed mathematical regression formulas where the root translucent dentin is considered as the only dental morphological parameter used for age estimation [5].

\section{Objectives}

The main goal of this research project is the validation of dental age estimation in a Portuguese adult population through those two dental methods, the radiological and the morphological, with the purpose of forensic applicability in age estimation in adult Portuguese cadavers.

\section{Materials and Methods}

To achieve the proposed objectives the research study was done in two distinct phases, a clinical and a laboratory phases. The clinical phase of this exploratory research and human teeth specimens came from samples extracted from alive persons for treatments reasons from the faculty of dentistry, university of Lisbon with informed consent of the patients. The laboratory phase consisted in the applicability of the direct and indirect methodology for age estimation.

\section{Experimental design from the pilot study}

This research work resulted from a pilot study carried out by the faculty of dentistry, university of Lisbon with the aim to analyz age estimation of the adult cadaver through the radiograph and morphological parameters in a portuguese adult population applied to single root teeth. The experimental protocol from this human investigation was approved and realized with the consent of ethics for health committee of the faculty. According to the experimental protocol given to all patients, an informed consent and the privacy of the data was assured.

\section{Population sampling}

The sample consisted of 35 teeth belonging to individuals from both genders aged over than 20 years. Data collection was made according to the extracted teeth from the patients from the oral surgery appointments from dental faculty, university of Lisbon performed by the dental students from the $4^{\text {th }}$ and $5^{\text {th }}$ year of the dental master course. The data collected are the number of individual clinical file, the chronological age on the day of extraction, the birthday data, the gender and the tooth identification. Tooth are separated in individual containers with a number from the project and stored in a solution of chloramines at a $4{ }^{\circ} \mathrm{C}$ to avoid the degradation of organic components of the dentin.

\section{Inclusion criteria}

In this research pilot study, the selection of the sample was based on the following inclusion criteria:

- Individuals over the age of 20 years

- Teeth without endodontic treatment

- No impacted teeth

- Teeth without crowns or without direct rehabilitation with radiopaque material in the vestibular surface

- Teeth without extensive abrasion injuries

\section{Methodology}

Non-invasive method: Radiographic analysis was performed according to the measurements proposed by Kvaal and Solheim [6] through the program of digital radiology software Kodak RVG intra-oral 2200 with exposure factors 60-70 KVp and $7 \mathrm{~mA}$, allowing dental radiographic images capture with a high-quality, a fast and an easy way. According to the tooth type the follow parameters were measured:

In the tooth:

- Length of apical dentin translucent of the tooth measured in millimeters ( $\mathrm{T})$.

- Retraction of the periodontal ligament measured in millimeters in the mesial surface of the root (LP).

In the digital radiographs:

- Length of pulp (CP).

- Root length measured from the mesial surface (CR).

- Pulp with at the cemento-enamel junction (LPJCE).

- Root width at the cemento-enamel junction (LRJCE).

- Pulp width at the midpoint of the length of the root (LPMR). 
- Root width at the midpoint of the length of the root (LRMR).

- The ratio of the length $(\mathrm{FL}=\mathrm{CP} / \mathrm{CR})$.

- The ratio of the width (FWC=LPJCE/LRJCE).

- The ratio of the width (FWM=LPMR/LRMR).

Invasive method: The analysis of dental morphological parameters was carried out on the intact and sectioned teeth. The objects of study were sectioned by a tungsten drill from the mesial side. The section was done on a vertical plane in the direction vestibule-lingual to expose the largest area of the pulp camera. The amount of translucent dentin was evaluated in millimeters from the apical end of the tooth into the coronal direction according to the Bang and Ramm methodology [9]. Measurements are calculated by a digital caliper Mitutoyo ${ }^{\oplus}$.

\section{Intra- and inter-observer calibrations}

In this research pilot study the measurements in radiological and morphological analysis are performed by the same observer. So we avoided introducing an inter-observer variability. However we assessed the intra-observer variability over time by measuring the intra-observer error.

The consist variability in intra-observer is due to involuntary data modification of the application and the criteria over time resulting in the introduction of systematic errors and/or random. This means that the same distance can be measured differently in different methods.

To prevent the introduction of systematic errors the methodological criteria was previously defined clearly. To deal with the random errors, in the validity of the data collected by the same investigator, observations were repeated in a period of time, long enough to eliminate the effect of memory. This period of time was one month and the maximum of repeated measurements are $10 \%$ of the total measurements. The results of the repeated measurements are compared with the first measurements and are obtained with the distribution of the two differences. Paired t-tests are used to calculate intraobserver differences.

\section{Statistical methodology of the analysis of the results}

The relationship between the coefficients is calculated between the age and the ratios. The chronological age is chosen as the dependent variable and the main components of the measurements as independent variables, the level of significance was $\mathrm{p}<0.05$. The chronological age corresponded to the individual's age in years at the time of extraction of tooth based on the date of birth registered in the clinical file of the patient. The dental age was calculated by each of the two methods defined at the specific objectives. The values obtained in measurements were analyzed through the Software program SPSS for Windows, version 18.0.

\section{Results}

The 35 tooth were carried out to digital peri-apical radiographs to evaluate the original Kvaal and Solheim method. The morphological parameter translucent dentin was determined in both intact and sectioned in order to assess the accuracy of the original method of Bang and Ramm in the adult Portuguese population. The descriptive analysis is carried out by statistical analysis, calibration and graphical representation. To test the accuracy a methodology is carried out. The Pearson's correlation coefficient of determination and analysis of variance ANOVA.

For dental age estimation we applied the mathematics regression formulas developed by Kvaal and Solheim in the digital periapical radiographs. These authors have obtained five regression formulas for each of the 10 dental group based on singles root teeth. To apply each of the regression formula, the measurements were estimated to calculate the ratios according to the previous definitions at the materials and methods.

By the method of Bang and Ramm we developed two mathematics regression formulas according to the length of translucent dentin were higher or lower than the $9 \mathrm{~mm}$.

The analysis of variance ANOVA, determined that there were no differences statistically significant between group means methodology. However, the Pearson's correlation coefficient (0.86) as well as the coefficient of determination (0.74) is the highest for the direct methodology for sectioned teeth and gives the best relationship between the dental age estimation and the chronological age in the adult Portuguese population (Table 1 and Table 2) with the smallest standard deviation (8.18 years) compared to the other assessed methods (Table 3 ). The result of the Pearson's correlation coefficient (0.89) is higher when we combined the two direct methods, in the intact and sectioned teeth, beside we applied isolated.

\begin{tabular}{|l|l|}
\hline Hypothesis & Pearson's correlation coefficient \\
\hline Radiograph Methodology & 0,832 \\
\hline $\begin{array}{l}\text { Morphology Methodology for } \\
\text { intact teeth }\end{array}$ & 0,819 \\
\hline $\begin{array}{l}\text { Morphology Methodology for } \\
\text { sectioned teeth }\end{array}$ & 0,863 \\
\hline $\begin{array}{l}\text { Morphology Methodology (intact } \\
\text { and sectioned) }\end{array}$ & 0,888 \\
\hline
\end{tabular}

Table 1: Pearson's correlation coefficient.

\begin{tabular}{|l|l|}
\hline Hypothesis & Coefficient of determination \\
\hline Radiograph Methodology & 0,692 \\
\hline Morphology Methodology for intact teeth & 0,670 \\
\hline $\begin{array}{l}\text { Morphology Methodology for sectioned } \\
\text { teeth }\end{array}$ & 0,744 \\
\hline $\begin{array}{l}\text { Morphology Methodology (intact and } \\
\text { sectioned) }\end{array}$ & 0,788 \\
\hline
\end{tabular}

Table 2: Coefficient of determination.

The males (29\% of total sample) had approximately a mean of standard error of 33.9 , while the females $(71 \%$ of the total sample) have 22.4. Based on these preliminary results, the Portuguese females have better results than the males (Graph 1, Table 4 and Table 5). 


\begin{tabular}{|l|l|}
\hline Hypothesis & Standard deviation (years) \\
\hline Radiograph Methodology & 9,040 \\
\hline Morphology Methodology for intact teeth & 8,419 \\
\hline $\begin{array}{l}\text { Morphology Methodology for sectioned } \\
\text { teeth }\end{array}$ & 8,180 \\
\hline $\begin{array}{l}\text { Morphology Methodology (intact and } \\
\text { sectioned) }\end{array}$ & 8,287 \\
\hline
\end{tabular}

The teeth that allowed better relationship between the real age and estimated age were the 33,43 and 11 .

\section{Discussion}

The aim of this pilot research study was to apply a radiographic method (indirect and non-invasive) and a morphological method (direct and invasive, destructive or non-destructive) to validate the original methods of Kvaal and Solheim and the Bang and Ramm at the adult Portuguese population for medico legal age estimation in adult cadavers.

The age parameter was defined for individuals with more than 20 years, since the methods proposed to test under the hypothesis are for age estimation in the adults group, where the physiological dental process are already finished. In our research project we did not set an upper bond on the age in inclusion criteria, since the sample is a representative of an ageing Portuguese population and represented by the patients who have been treating in the clinical services from the dental faculty. However, according to some studies there is a higher variation in age estimation results in individuals aged over 60 years [12].

About $8 \%$ of the total sample obtained the age estimated equal to the real age. The percentage of the results obtained with underestimated age (between -4 and -6 years and between -7 and -9 years than the real age) are the same obtained with overestimated age (between 4 and 8 years than the real age), $46 \%$ for both genders.

The age of the individuals with less than 60 years tend to be overestimating instead of adults over than 60 years, who tend to be underestimating (Graph 2).

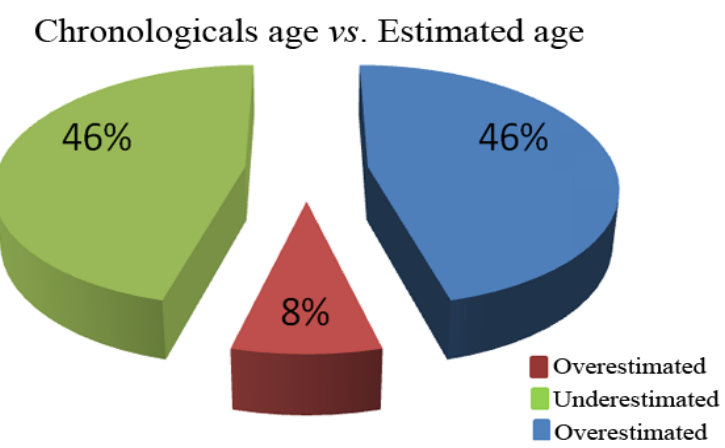

Graphic 2: Relation between estimated age and chronological age.

The majority of published methods for dental age estimation in a cadaver require tooth extraction (destructive technique). However, some forensic circumstances may be considered unacceptable. Such as the forensic cases where ethical, religious, cultural or scientific reasons are an imposition [2]. Due to these problems the radiographic techniques are considered as an advantage so far as they correspond to a simple and non-invasive method that can also be used on living individuals as well as cadavers [13]. It is important to note that there is a limitation of $\mathrm{x}$-ray films related to twodimensional images and to low resolution.

Radiographic analysis was done by digital radiology system that allowed dental radiographic images capture with highquality, a minimum degree of distortion, a quickly and an easily way and as well as allowed the investigators to do the measurements from the hypothesis on a way more accurate [14]. The use of digital periapical radiographs according to Willems and others authors; allow the realization of radiographic images more calibrated in such a way that the linear measurements are more accurate [14].

\begin{tabular}{|l|l|l|l|l|}
\hline & Radiograph Methodology & $\begin{array}{l}\text { Morphological Methodology for sectioned } \\
\text { teeth }\end{array}$ & $\begin{array}{l}\text { Morphological Methodology } \\
\text { from for intact teeth }\end{array}$ & Average \\
\hline Female sample size & 25 & 25 & 25 & 25 \\
\hline Total diference & 16,5 & 11,05 & $-27,86$ & $-0,13$ \\
\hline Average & 0,66 & 0,442 & $-1,1144$ & $-0,0052$ \\
\hline
\end{tabular}

Table 4: Residuals analysis of regression analysis from female population.

\begin{tabular}{|l|l|l|l|l|}
\hline & Radiograph Methodology & $\begin{array}{l}\text { Morphological Methodology for sectioned } \\
\text { teeth }\end{array}$ & $\begin{array}{l}\text { Morphological Methodology } \\
\text { from for intact teeth }\end{array}$ & Average \\
\hline Male sample size & 10 & 10 & 10 & 10 \\
\hline Total diference & 24,2 & 21,35 & -1 & 14,85 \\
\hline Average & 2,42 & 2,135 & $-0,1$ & 1,485 \\
\hline
\end{tabular}

Table 5: Residuals analysis of regression analysis from male population. 
The inclusion criteria are the criteria for including a patient in the study, and it is important that these criteria be clearly defined in an objective manner, they were teeth without: endodontic treatment, crowns or vestibular radiopaque restorations and abrasion lesions [4]. They are co-variables and the methodologies choose for testing the main hypothesis which couldn't be answer.

The sample from this pilot research were included all single root teeth of the upper and lower jaws, central and lateral incisors, canine, first and second premolars. The multiradiculares teeth were excluded from this research study due to the difficulty in defining the pulp area associated with each root at the radiography. For statistical analysis we established a linear relationship between the dependent variable (real age) and the independent variables (age estimation by the morphological and radiographic parameters) [15]. In the process of the measurements, there might have small inaccuracies such as a slight tooth inclination relative to the direction of the radiographic funnel. The largest source of error of the measurements was the difficulty in identifying the points of reference in $\mathrm{x}$-rays when viewed on a monitor. When analyzed a tooth for each person, what often occurs in a forensic investigation research, we must calculate the standard deviation as well as the confidence interval in age estimation.

Some authors claim that the difference between the real length of the teeth and the length measured in digital $\mathrm{x}$-rays is statistically insignificant when we paralelometric radiographic technique [4]. In this research the $\mathrm{x}$-rays were carried out according to this methodology to minimize the variance of the lengths of the measurements. The statistical analysis of variance ANOVA testing has determined that there are no significant statistically differences between the methods. Therefore, these methods may be applied for dental age estimation in the adult cadaver Portuguese population.

The results obtained in this pilot research study in the Portuguese population, where the radiograph method had the lower Pearson correlation coefficient (0.83) (Graph 3) as well as the lower coefficient of determination (0.69), are in agreement with the conclusions from other scientific researches.

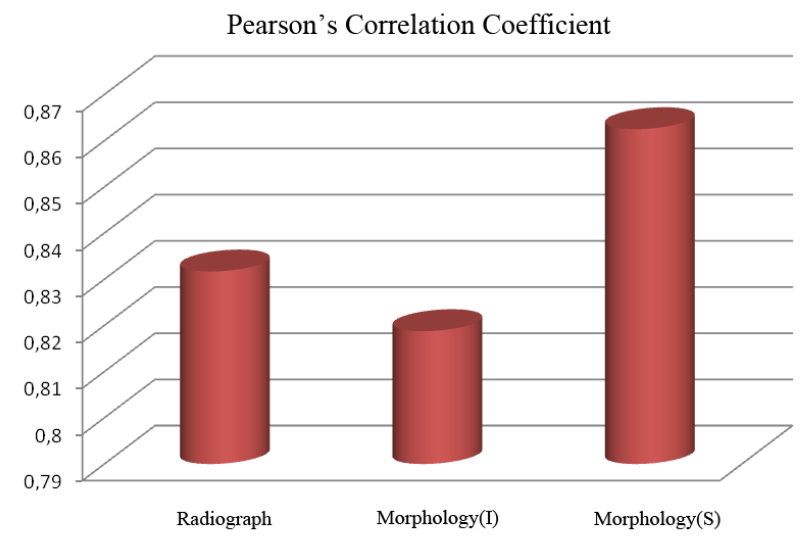

Graphic 3: The Pearson correlation coefficient for the Portuguese population obtained with the three methods: Radiograph, Morphology for non-sectioned teeth (I) and Morphology for sectioned teeth (S).
The Pearson correlation coefficient $(0.86)$ as well as the coefficient of determination (0.74) for the adult Portuguese population allows us to conclude that the direct method for sectioned teeth gives the better relationship between the real age and the estimated age than radiograph method.

The original Bang and Ramm method for sectioned teeth presented an average for standard deviation of 10.87 years [16]. In our research study, for all assessed methods, the standard deviation was lesser. The morphological method for sectioned teeth had the best standard deviation for an adult population of 8.18 years (Graph 4 ).

Translucent dentin vs. age

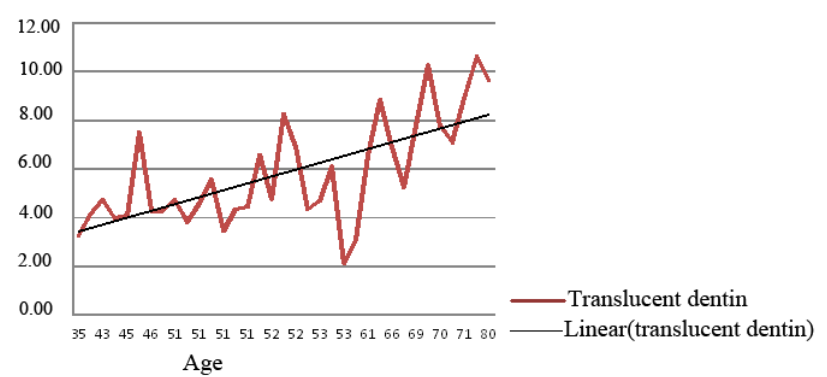

Graphic 4: Relationship between the length of the translucent dentin and the chronological age.

The variance obtained by different methods, about $8 \%$ of the sample obtained an estimate of age equal to real age. The old underestimated and overestimated corresponds respectively to $46 \%$ of the sample. The largest percentage of overestimated age corresponds to a smaller range of years, 6 years (4 to 23\%). The old underestimated had the same percentage (17\%) for the ranges -6 to -4 years and -7 to -9 years. The sample had $71 \%$ females and $29 \%$ males. The average error for males had a mean of approximately equal to 33.9, while the females have 22.4. This conclusion is in disagreement with the study of star and others, who points out that the estimated age is independent of gender [17].

In a second statistical analysis we created two groups from the total sample of 35 teeth for each group of gender, one with individuals aged less or equal than 60 years and the other group from the individuals with more than 60 years. This statistical analysis was important according to the differences between age estimation less than 60 years old and over 60 years old. The females aged less or equal than 60 years obtained a highest standard deviation in the range of 1 to 3 years of overestimated age $(20 \%)$. For female with more than 60 years the greatest standard deviation occurred in the range of -7 to -9 years of underestimated age (9\%). Different results were obtained for the males group, where individuals with age equal or less than 60 years had a greater standard deviation in the range of 4 to 6 years of overestimated age (9\%). For males with more than 60 years were obtained equal results in the ranges - 4 to -6 years and -7 to -9 years of underestimated age (9\%).

The Bang and Ramm has a tendency to overestimate the age when we analyzed teeth from young adults and underestimate age in the group of older age [16]. In our research project we obtained for Portuguese population the same results.

The cutting technique used was chosen over others, because this allows getting better results on the age-related dental changes through the measurements performed for the parameter of tra- 
nslucent dentin [18]. The length of the translucent dentin for sectioned teeth had a greater correlation with the age estimation when compared with intact teeth, according to other investigations [14]. This is related to the measurement of the translucent dentin on the intact teeth correspond a three-dimensional evaluation, which means the variable is lesser accurate, when the translucent dentin is evaluated in the sectioned teeth, a two-dimensional analysis [10]. The results obtained in this adult Portuguese study reinforced this assumption, the evaluation of the translucent dentin in sectioned teeth allowed best age estimation.

\section{Conclusions}

This forensic pilot research allowed us to evaluate the applicability of two dental age estimation approaches, a radiograph and a morphological in an adult Portuguese population for age estimation in an adult cadaver. Based on the results of this study, it can be concluded that both nondestructive and destructive dental-age estimation techniques were able to produce significant accurate dental age estimations, at least when these techniques were applied appropriately. However, to the forensic deontologists recommended to use different age estimation techniques and perform repetitive measurements in order to verify the reproducibility of the calculations performed. The main objective is to obtain the best standardized method for legal medical age estimation, reproductive, simple and reliable that can be applied in cadavers from Portugal in the field of human identification.

\section{Acknowledgements}

Research partially sponsored by national funds through the Fundação Nacional para a Ciência e Tecnologia, Portugal FCT under the project (PEst-OE/MAT/UI0006/2011).

\section{References}

1. Brkic H, Milicevic M, Petrovecki M (2006) Age estimation methods using anthropological parameters on human teeth-(A0736). Forensic Sci Int 162: 13-6.

2. Kvaal SI, Kolltveit KM, Thomsen IO, Solheim T (1995) Age estimation of adults from dental radiographs. Forensic Sci Int 74: 175-85.

3. Garamendi PM, Landa MI, Ballesteros J, Solano MA (2005) Reliability of the methods applied to assess age minority in living subjects around 18 years old. A survey on a Moroccan origin population. Forensic Sci Int 154: 3-12.
4. Kolltveit KM, Solheim T, Kvaal SI (1998) Methods of measuring morphological parameters in dental radiographs. Comparison between image analysis and manual measurements. Forensic Sci Int 94: 87-95.

5. Meinl A, Huber CD, Tangl S, Gruber GM, Teschler-Nicola M, et al. (2008) Comparison of the validity of three dental methods for the estimation of age at death. Forensic Sci Int 178: 96-105.

6. Kvaal S, Solheim T (1994) A non-destructive dental method for age estimation. J Forensic Odontostomatol 12: 6-11.

7. Knell B, Ruhstaller P, Prieels F, Schmeling A (2009) Dental age diagnostics by means of radiographical evaluation of the growth stages of lower wisdom teeth. Int J Legal Med 123: 465-9.

8. Willems G (2001) A review of the most commonly used dental age estimation techniques. J Forensic Odontostomatol 19: 9-17.

9. Bang G, Ramm E (1970) Determination of age in humans from root dentin transparency. Acta Odontol Scand 28: 3-35.

10. Solheim T (1989) Dental root translucency as an indicator of age. Scand J Dent Res 97: 189-97.

11. Acharya AB, Vimi S (2009) Effectiveness of Bang and Ramm's formulae in age assessment of Indians from dentin translucency length. Int J legal Med 123: $483-8$.

12. Willems G, Moulin-Romsee C, Solheim T (2002) Non-destructive dentalage calculation methods in adults: intra- and inter-observer effects. Forensic Sci Int 126: 221-6.

13. Landa MI, Garamendi PM, Botella MC, Aleman I (2009) Application of the method of Kvaal et al. to digital orthopantomograms. Int J Legal Med 123: 123-8.

14. Sharma R, Srivastava A (2010) Radiographic evaluation of dental age of adults using Kvaal's method. J Forensic Dent Sci 2: 22-6.

15. Olze A, Mahlow A, Schmidt S, Wernecke KD, Geserick G, el at. (2005) Combined determination of selected radiological and morphological variables relevant for dental age estimation of young adults. Homo 56: 133-40.

16. Soomer H, Ranta H, Lincoln MJ, Penttila A, Leibur E (2003) Reliability and validity of eight dental age estimation methods for adults. J Forensic Sci 48: 149-52.

17. Star H, Thevissen P, Jacobs R, Fieuws S, Solheim T, et al. (2011) Human dental age estimation by calculation of pulp-tooth volume ratios yielded on clinically acquired cone beam computed tomography images of monoradicular teeth. J Forensic Sci 56 : S77-82.

18. Solheim T (1984) Dental age estimation. An alternative technique for tooth sectioning. Am J Forensic Med Pathol 5: 181-4.

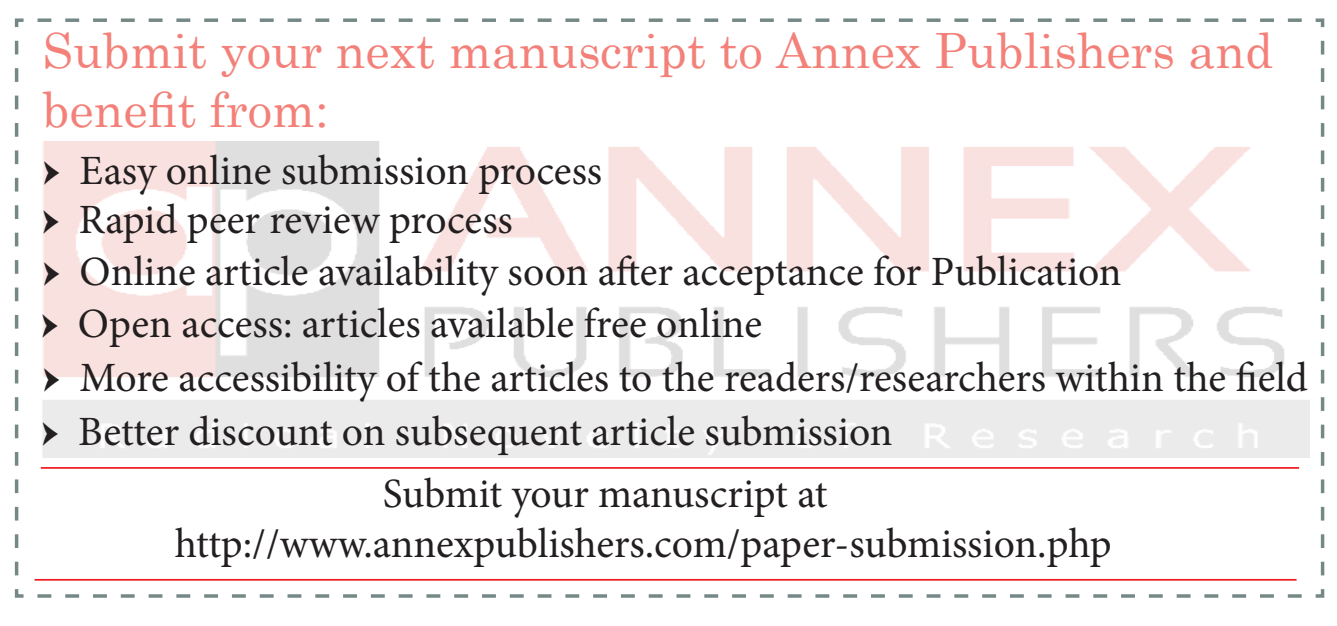

